\title{
An experimental study of exploitation of materials used for prosthetic temporary restorations
}

\author{
Leszek Szalewski 1,*, Elżbieta Celej-Piszcz¹, Matgorzata Stodółkiewicz², Radostaw Cechowicz ${ }^{3}$, Marcin Bogucki \\ Janusz Borowicz ${ }^{1}$, and Jacek Szkutnik ${ }^{1}$ \\ ${ }^{1}$ Medical University of Lublin, Department of Dental Prosthetics, Karmelicka 7, 20-081 Lublin, Poland \\ ${ }^{2}$ Medical University of Lublin, Department of Functional Masticatory Disorders, Karmelicka 7, 20-081 Lublin, Poland \\ ${ }^{3}$ Lublin University of Technology, Mechanical Engineering Faculty, Department of Automation, Nadbystrzycka 36, 20-618 Lublin, \\ Poland
}

\begin{abstract}
Temporary restorations more often play their role for a definitely longer period of time, than the time necessary for implementation of final prosthetic work. Therefore, they are subjected to adverse effects of chemical, physical and thermal factors in a patient's oral cavity. Since loss of temporary prosthetic work can have negative consequences for an entire treatment process, it is important to identify a potential risk of damaging temporary restorations by factors derived from the diet of patients. The aim of this study was to evaluate the influence of components of the beverages, such as coffee and tea, consumed daily by the patients, on the mechanical properties of materials used in temporary restorations. The experimental design applied, as well as gained results, were justified by appropriate, chosen statistical methods. The conducted research demonstrated the change of mechanical properties of studied materials for their flexural strength, as well as microhardness. The tendency of changes in both result variables, compared with the samples kept in neutral environment - distilled water, indicates the decrease in mechanical strength and microhardness of materials conditioned in coffee and tea. It can be claimed that the components of beverages present in an everyday diet of patients have a considerably adverse effect on the quality of materials used for provisional crowns and bridges.
\end{abstract}

\section{Background}

New possibilities of prosthetic treatment sometimes require fixed temporary restorations to be used for longer than the time needed to prepare a final restoration.

During treatment, patients expect temporary restorations to be esthetic and comfortable thus, the requirements for temporary restorations are almost identical with those set up for final restorations [1]. Additionally, apart from being esthetic, temporary restorations must meet several conditions - protection of a living dental pulp against chemical, thermal and mechanical factors, assurance of stable occlusion, continuity of functions of stomatognathic system and support for soft tissues [2].

Currently, on the market, there are many materials for temporary prosthetic restorations. These are mainly materials based on acrylic mass or composite materials, and they can meet requirements for temporary restorations to a different extent.

A prosthesis in the oral cavity is constantly prone to mechanical, chemical and thermal factors deriving from the diet of patients. These factors can considerably influence mechanical and esthetic properties of prepared crowns and bridges [3]. A human should drink approximately 2-3 liters of liquids. The type of liquid depends on the age and individual patient's preferences.
The adults, who constitute the majority of patients treated prosthetically, most often drink coffee and tea. These beverages can have a crucial impact on mechanical and esthetic properties of temporary and final restorations.

The materials for temporary restorations can change their mechanical and esthetic properties. Diaz-Arnold et al. found that the microhardness of 5 different temporary materials diminishes with time, regardless of chemical or thermal factors derived from the patient's diet [4]. In their studies, they kept samples in artificial saliva for 14 days and after that time they compared microhardness with primary samples measured after 24 hours since preparation. On the other hand, Balkenhol et al. while comparing the flexural strength, depending on the time that passed from polymerization, proved that for the first few hours materials showed lower flexural strength [3]. The effect can be explained by the fact that materials prepared on the basis of acrylic products need more time to undergo full polymerization. When comparing materials based on composites and acrylic resins, Balkenhol suggested the use of composite materials, which are characterized by higher values of flexural strength directly after their preparation [5]. The analysis of literature studies, related to the effect of dietary factors on the properties of materials used for temporary restorations, indicates several articles concerning the change of color of temporary restorations under the

\footnotetext{
*Corresponding author: leszek.szalewski@,umlub.pl
} 
influence of exogenous coloring agents [1,6,7]. Yet, there are few studies determining the influence of dietary factors on the mechanical properties of temporary materials. Akova et al. evaluated an influence of heptane, ethanol and citric acid on the mechanical properties of temporary material samples [8]. When analyzing the subject literature, publications describing the influence of beverages consumed by patients with temporal prosthesis, such as coffee, tea, fruit juices and alike, have not been found. Thus, it is highly justified to conduct such studies. An experimental design and statistical analysis of its outcomes could validate the expected premises about detrimental influences of the aforementioned factors. Moreover, results gained from this study might contribute to defining the recommendations for patients using temporary restorations, especially for a long period of time.

\section{Objectives}

The aim of this study was to evaluate the influence of components of the beverages, such as coffee and tea, consumed daily by patients on the mechanical properties of materials used in temporary restorations. In the research, an experimental design of univariate analysis was applied. The studied factors were compared with the influence of substances which are contained in coffee and tea; yet, each of the factors was studied individually.

The effects of interactions (in other words: crossvalidation) of simultaneous interaction of two factors were not included in the study. The impact of the studied factors on the temporary restoration material was examined for three agreed systems. The first experimental system was treated as so-called control group and it corresponded to the samples of conditioned materials in a neutral environment. The rest of two experimental systems were related to the conditioning of samples in two water solutions respectively: coffee and tea. The studies tried to prove the effect of change in the mechanical properties of materials, namely flexural strength and microhardness under the influence of substances from the two solutions mentioned above.

Altogether, there were six experiments conducted during the study - two for each three tested materials used for temporary restorations.

\section{Material and methods}

Three materials for temporary restorations available on the market were used in the study: Structure 2 (VOCO), Unifast TRAD (GC) and Revotek LC (GC). 24 samples in the shape of a cuboid measuring $2 \times 2 \times 25 \mathrm{~mm}$ and 30 disc-shaped samples measuring $5 \times 2 \mathrm{~mm}$ were made from each of the materials. The samples were prepared in specially designed metal matrices. The metal form was layered thinly with vaseline, to avoid the material sticking to the matrix. The material was condensed with hand pluggers. Each sample, requiring polymerization with the use of light, was treated with radiation from LED Clear Blue lamp of $1200 \mathrm{~mW} / \mathrm{cm}^{2}$. Both sides of every sample were radiated for 20 seconds. The samples were polymerized via polyethylene foil, to eliminate the oxygen inhibition layer. After hardening, the samples were cautiously taken out of the matrix, so that the edges stayed intact. Possible uneven parts were polished with the Sof-lex (3M ESPE) disc. Then, the samples were thoroughly examined to eliminate samples with air bubbles or other inaccuracies that might have emerged during condensation and polymerization. For the first 24 hours, the samples were kept in distilled water in $370 \mathrm{C}$. After that time, the samples were randomly divided into three subgroups and conditioned in fluids: coffee (Nescafe), tea (Lipton) and distilled water for 7 days. The coffee solution was prepared by pouring 2 spoons $(10 \mathrm{~g})$ of instant coffee Nescafe Classic with $200 \mathrm{ml}$ of boiling water. On the other hand, the tea solution was made from 1 tea bag of Lipton Yellow Label Tea poured over with $200 \mathrm{ml}$ of boiling water and left for 5 minutes. Next, the samples were rinsed with distilled water and dried. Then, they underwent the flexural and Vickers microhardness tests.

\subsection{Flexural testing}

Flexural testing was conducted with the use of materials testing machine (Zwick Roell) in the support spacing $20 \mathrm{~mm}$ and the speed of head $0,75 \mathrm{~mm} / \mathrm{min}$, with the initial load of $1 \mathrm{~N}$. The width and thickness of every sample was measured with the use of a digital caliper to obtain possibly the most accurate results. The test finished when a sample was broken.

The tension determining the flexural strength was calculated on the basis of the following formula:

$$
\sigma=3 \mathrm{~F}_{\mathrm{m}} \mathrm{I} /\left(2 \mathrm{bh}^{2}\right)
$$

where:

$\mathrm{F}_{\mathrm{m}}$ - the maximum load applied at the moment of a sample braking,

I - the support spacing $10 \mathrm{~mm}$,

$\mathrm{b}$ - the width of a sample,

$\mathrm{h}-$ the thickness of a sample.

\subsection{Vickers microhardness study}

In case of measuring microhardness of provisional restorative materials, the Vickers test is better than other methods of examining hardness, due to its low sensitivity to the material surface imperfections after polymerization. The microhardness testing was conducted with the Vickers method with the use of Micro Hardness Tester mhp 100 and the load of $20 \mathrm{~g}$ for 10 seconds.

The length of the diagonal imprint on the surface of a studied sample was measured by examining sample surface in the optical microscope Neophot 2. Each sample had 3 imprints done in different areas of its surface. While calculating, it was considered that the microhardness is proportional to the quotient of the load and the imprint surface area, which is assumed to have a shape of a regular pyramid with a square base and the same apex angle as its indenter. 


\subsection{Statistical analysis of the study results}

The series of result variables measurements underwent the preliminary analysis with the exclusion of extreme values, which were observed in the case of flexural testing. The extreme values occurred due to the unrepeatable sample setting in the material testing machine and the impurity (defective structure) of a sample material. The measurement of result variables compared to the rest of experimental samples, subjected to the same environmental impact, demonstrated only random changes in an established mean value, which indicated maintaining repeatable study conditions.

The conducted research was analyzed statistically with the use of the following experimental data analysis [9]:

- Parametric variance analysis, when the results of trials observed in given experimental systems were characterized by homogenous variance, and when the distribution of the rest of the variance model was not different from the normal distribution, which was verified by an appropriate statistical testing;

- Non-parametric type of variance analysis - the rankbased Kruskal-Wallis test when the above-mentioned assumptions were not met;

- Post hoc comparison tests of the measures achieved in given experimental systems, which aim was to indicate reciprocal, statistically significant differentiation of the results in the range of the used restoration material. In the case of the parametric analysis, the HSD Turkey test was applied, otherwise, an appropriate type of nonparametric test was used.

The aim of the ANOVA analysis was to demonstrate the negative impact of dietary factors contained in the studied beverages. Due to the conciseness of the research paper, only the most significant results and comments of statistical analyses were enclosed in the publication Box-Plot figures summarizing descriptive statistics of measurements achieved in given experimental systems (mean value with the standard error - illustrated with socalled figure frame and the highest and lowest value of measurement - so-called whiskers). In every statistical test, the probability $\alpha=0.05$ was assumed as a threshold of the statistical significance.

\section{Results}

The measurements of the result variables: microhardness and flexural strength have been presented in Figs. 1 and 2.

The conducted research demonstrated the change of mechanical properties of the studied materials for their flexural strength, as well as microhardness. The tendency of changes in both result variables, compared with the samples kept in neutral environment - distilled water, indicates the decrease in mechanical strength and microhardness of materials conditioned in coffee and tea. The discussed changes are visible in Box-Plot figures (1 and 2), which was formally proven by the analysis of variation results and the results of post-hoc comparisons. In the case of flexural strength of materials, none of the experimental trials conducted for control group samples demonstrated variation in the level of mean values which was approximately $66 \mathrm{MPa}$.

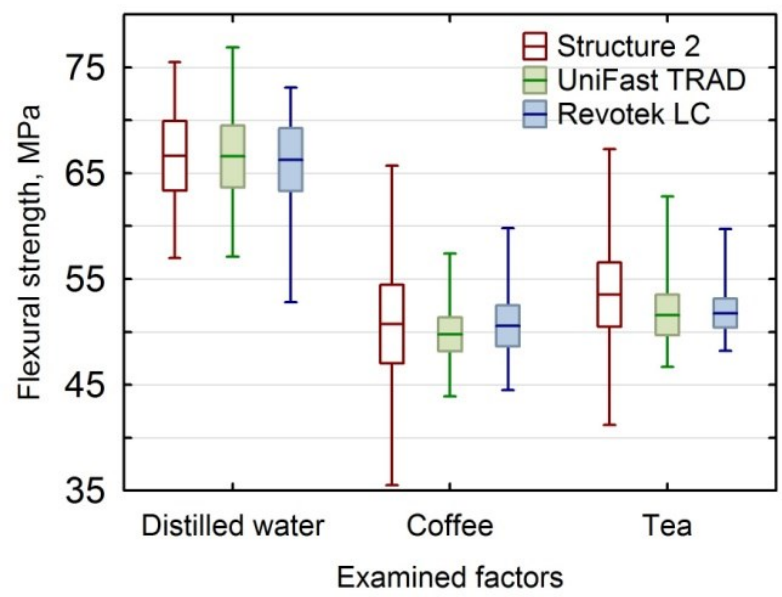

Fig. 1. The summary of flexural strength measurement results achieved in given experimental sets

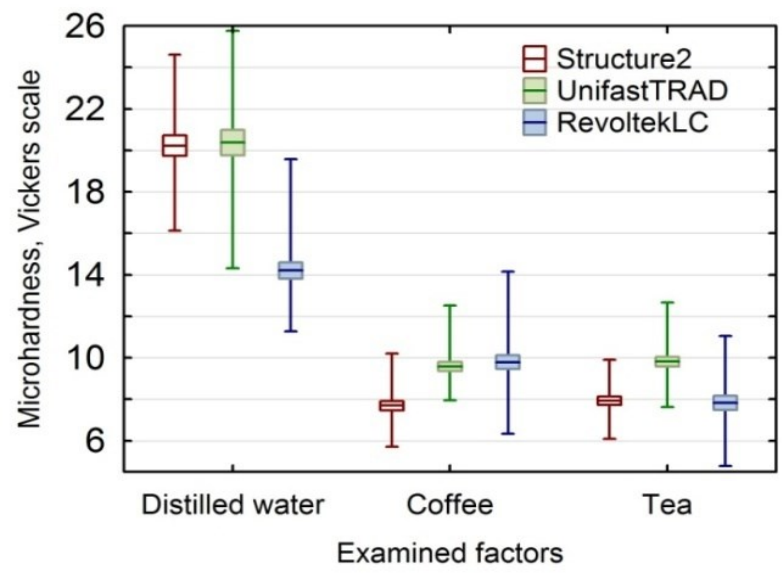

Fig. 2. The summary of microhardness measurement results achieved in given experimental sets

Similarly, statistically significant differences in the measurements of the flexural strength of the samples made from various materials conditioned in coffee or tea solution were not observed. This result proves a very similar influence of the studied solutions on the temporary restorations. The post-hoc tests showed that the influence of coffee solution components on the samples made from various materials was not considerably different from the impact of the tea solution ingredients, in a statistical and practical sense. The above-mentioned observations concern also the second group of studied variables - microhardness.

\section{Discussion}

It should be noted, that the 3-point bending test and Vickers microhardness test were used in the research. Flexural strength measured with 3-point bending test reflected processes occurring in the oral cavity, where the prosthetic bridge is supported by abutment teeth on both edges, and the destructive force occurs in the middle of restoration, in the span of a bridge.

In the studies, the samples were immersed in the tested fluids for 7 days, which seems to be a long period. 
Despite the fact, that restorations in an oral cavity are constantly cleaned by saliva or hygienic procedures, the food can remain in unavailable places (such as overhangs of temporary prosthetic works or under the spans of the bridge) for a long time. The adverse factors derived from the diet of patients can also be absorbed by plaque and tartar, which additionally extends the contact of chemical factors with teeth and restorations.

All the materials tested in the control samples were characterized by the similar average flexural strength $(66 \mathrm{MPa})$ and in the case of microhardness, the lowest value was achieved by Revotek material. This may be due to a lower filler content (15-35\%), as compared to conventional composite materials with a filler content of $85 \%$ [8].

The microhardness of the samples after immersing in beverages decreased by approx. 50-60\% for Structure 2 and Unifast TRAD materials, and approx. $35 \%$ for Revotek material. The observed effects can be explained by the lack of material surface polishing, which increases the value of microhardness of the material and resistance to chemical agents [7].

Similar results of the negative impact of food onto the materials used for prosthetic temporaries were achieved by Akova et al. [8], in the case of heptane and $75 \%$ ethanol use. Just in the case of 0,02 citric acid, the increase in Knoop microhardness values was observed. Analogous results were depicted in the studies concerned with the composite materials used for direct teeth restoration $[10,11]$.

There are few papers describing the impact of diet on temporary restorations in the oral cavity; as it is commonly believed, because of a short period of use. However, due to diagnostic reasons, temporary restorations more and more often must remain in the oral cavity for a longer period (even for a few months). Hence, it seems important to examine what impact the chemical and/or thermal factors derived from the diet of patients can have on this kind of restorations.

\section{Conclusions}

Taking the results of the discussed studies into account, it can be claimed that the components of beverages present in the everyday diet of patients have a considerably adverse effect on the quality of materials used for provisional crowns and bridges. Thus, dietary recommendations for patients using temporary restorations for a longer period and the diligence of their preparation should be emphasized. Provisional restorations, as well as fixed restorations, cannot encourage the stagnation of food and should be available for self-cleaning and hygienic procedures. The durability of temporaries is also affected by the choice of material with suitable properties, which should be the subject of further experimental research.

\section{References}

1. D.K. Prasad, H. Alva, M. Shetty: J. Ind. Prost. Soc., 14, 1 (2014)

2. S. Rosenstiel, M.F. Land, J. Fujimoto: Contemporary Fixed Prosthodontics (Elsevier Health Sciences, 1141, 2006)

3. M. Balkenhol, P. Ferger, M. Mautner, B. Wostmann, Dent. Mater. 23, 12 (2007)

4. A.M. Diaz-Arnold, J.T. Dunne, A.H. Jones, J. Prosthet. Dent. 82, 5 (1999)

5. M. Balkenhol, M.C. Mautner, P. Ferger, B. Wostmann, J Dent. 36, 1 (2008)

6. M.D. Gross, J.B. Moser, J. Oral Rehabil. 4, 4 (1977)

7. Y.K. Lee, J.M. Powers, J. Biomed. Mater. Res. Part B Appl. Biomater. 73, 2 (2005)

8. T. Akova, A. Ozkomur, H. Uysal, Dent Mater, 22, 12 (2006)

9. D. Montgomery, Design and Analysis of Experiments, (John Wiley and Sons, 2004)

10. H. Sazak-Öveçoğlu, M. Günday, M.L. Öveçoğlu, F. Özer, B. Tarçın,. K. Eng. Mater. 264-268 (2004)

11. A.U. Yap, J.S. Low, L.F. Ong, Oper. Dent. 25, 3 (2000) 